\title{
Knowledge Based Enterprise Engineering (KBEE): A Modeling Framework for Enterprise Knowledge Capitalization
}

\author{
Mahmoud Moradi, Mounir Badja, and Bruno Vallespir \\ IMS-LAPS/GRAI, Bordeaux University, CNRS, \\ 351 cours de la Libération, 33405 Talence cedex, France, \\ \{Mahmoud.Moradi, Mounir.Badja, Bruno.Vallespir\} @ims-bordeaux.fr
}

\begin{abstract}
The main objective of this paper is to study the complementarity nature of enterprise modeling and knowledge management within the framework of enterprises. To do so, we evaluate some important methodologies in each domain and then several modeling points of view are explained. By reviewing CommonKADS, MASK, CIMOSA, and GIM methodologies and their modeling views we propose a generic framework to model knowledge in all its aspects. This framework has four modeling views namely, intent modeling, context modeling, content modeling and evolution modeling. These views are classified into several sub-elements brought together by developing meta-model in UML class diagrams. KBEE may possibly be useful both for the practitioners and scientific to deal with knowledge and its modeling in enterprises.
\end{abstract}

Keywords: Enterprise modeling, Knowledge modeling, Enterprise Engineering, Knowledge Engineering.

\section{Introduction}

Knowledge is widely recognized as being the key capital of enterprises that contributes to enterprise competitiveness and provides the basis for long term growth, development and existence. Therefore, one of the major questions is how to make more efficient use of knowledge in the enterprise (in terms of sharing or acquiring). It is a well known fact that much of existing information and knowledge, which is extremely valuable, is not made explicit, externalized or formalized and is consequently not available for use by other individuals, and sometimes it can even be lost for the enterprise. Therefore, how can informal enterprise knowledge be captured, formalized, organized, spread and reused?

From the hypothesis that modeling of knowledge and enterprise are imperative tools to manage knowledge in the organizations especially in externalization and integration of knowledge and also for the engineering context, we will propose in this paper a method for engineering the enterprise based on knowledge. This method is a result of synthesis of four main frameworks in Enterprise Modeling (EM) and Knowledge Management (KM). This method put in a shared context approximately all of the aspects concerning knowledge in the enterprises and looks at the knowledge from 
several point of views and entitled KBEE (Knowledge Based Enterprise Engineering). For the comparison of methods we propose a framework to classify all views into four mains categories as context modeling, intent modeling, content modeling, and evolution modeling. These components together are the basic foundation of reviewed methods. KBEE is a model based enterprise engineering framework that aims at modeling knowledge in all shapes and all sources in the enterprises in the perspective to provide an integrated and multifaceted knowledge base.

As a starting point, we draw on the CommonKADS, MASK, CIMOSA, and GRAI/GIM methodologies which all are intended for supporting and representing several views to model enterprises. These methods together provide an integrated view in enterprise context modeling (Fig. 1).

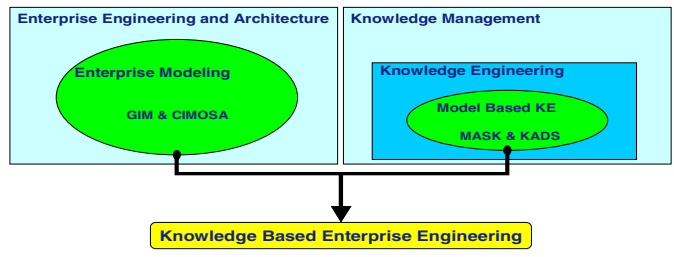

Fig. 1. Theoretic Foundation

\section{Meta-modeling}

Meta-modeling is the act of modeling applied to languages. The study of intersections between languages for their unification is largely facilitated by meta-modeling. Accordingly, we will use a meta-modeling approach to describe the selected enterprise modeling and knowledge management methods. Each method must be defined through a meta-model. In addition, the meta-modeling language being used is the UML class diagram [1] because it seems sufficient to deal with our problem which is, mainly, to describe the conceptual aspects of the methods. Indeed, for each method, a meta-model is built with a class diagram. With these meta-models, we can compare the constructs of the various methods.

However, it is important to note that the meta-models which we want to build here are not meta-models on the software science point of view. Thus, these meta-models have to be understandable for analysts of the enterprise modeling domain. They have to be clear and not necessarily to be the "best" meta-models, optimized and with the lowest number of classes. Moreover, meta-models are not comprehensive model of methods. This means meta-models developed here are approximately completed ones. Last point about meta-models is that they do not cover all the concepts and formalisms exist in the methods. We use simplified meta-model for the reason which it will cover basic concepts and elements.

\section{Knowledge Modeling and Enterprise Modeling Techniques}

From modeling point of view, the tools and techniques that help to model knowledge in the enterprises context are the main approaches to share and transfer knowledge. In 
one part the CommonKADS knowledge engineering methodology and MASK method are two methods that help knowledge sharing and transfer by applying knowledge engineering and knowledge capitalization tools with developing a number of formalisms. In another part CIMOSA and GRAI/GIM methodology are two wellknown enterprise modeling framework with several views and formalisms. A point should to be taken into account is that we did not choose these methods for the reason by which they are comprehensive methods in the field.

CommonKADS views knowledge engineering as a modeling activity, where each model is "a purposeful abstraction of some part of reality" [2]. Each model focuses on certain aspects of the knowledge and ignores others. It proposes six models at successively deeper levels of detail: knowledge engineers are encouraged to model the organization in which the system will be introduced; the task (business process) which the Knowledge Based System (KBS) will support; the agents who are or will be involved in the process; required communication between agents during the process; the knowledge which is applied to performing the knowledge-based process; and the design of the proposed KBS. Fig.2 illustrates meta-model of views of CommonKADS.

MASK (Method of Analysis and Structuring Knowledge) offers a flexible environment that allows to success knowledge capitalization projects [3] and [4]. Modeling tools as defined in MASK are generic enough and respond to expert's workspace and cognitive vision. These tools have an important role in knowledge extraction and formalization. They help to structure knowledge under: systemic, ergo-cognitive, psycho-cognitive, historical, and evolution views [5]. These views together construct knowledge modeling in MASK. Detail meta-model of views in MASK is depicted in fig. 3.

CIMOSA (CIM Open System Architecture) provides guidelines, architecture and an advanced modeling language for enterprise modeling covering several aspects of the enterprise [6]. The concept of views allows working with a subset of the model rather than with the complete model providing especially the business user with a reduced complexity for his particular area of interest. CIMOSA has defined four different modeling views: Function, Information, Resource and Organization [7]. See fig. 4 .

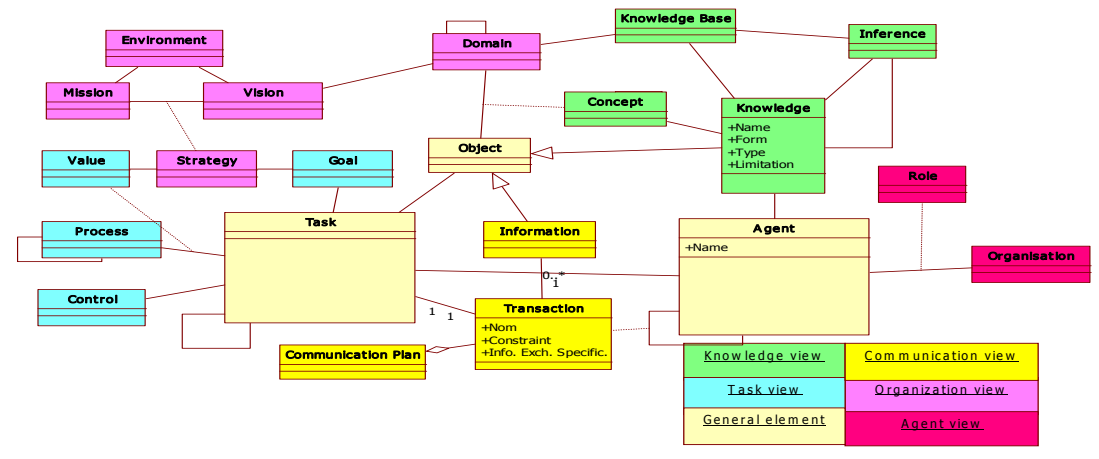

Fig. 2. Meta-model of CommonKADS 


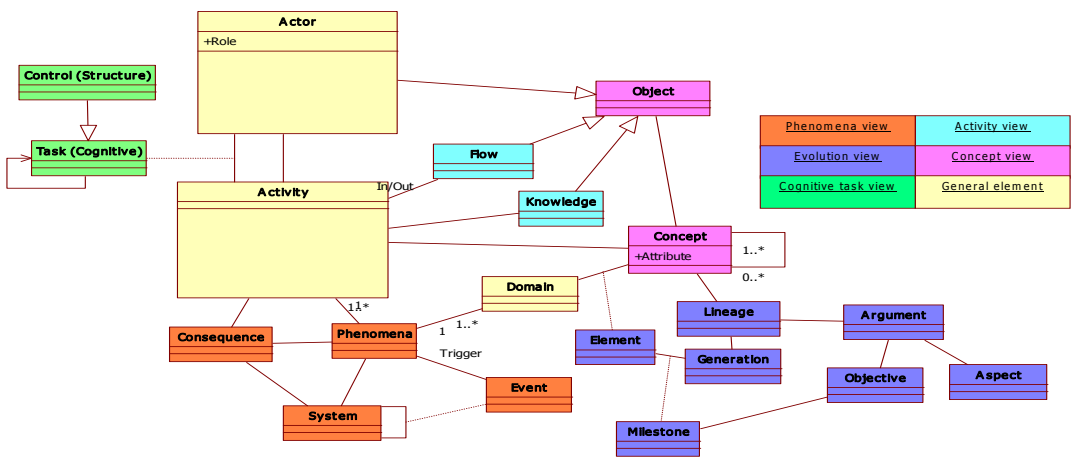

Fig. 3. Meta-model of MASK

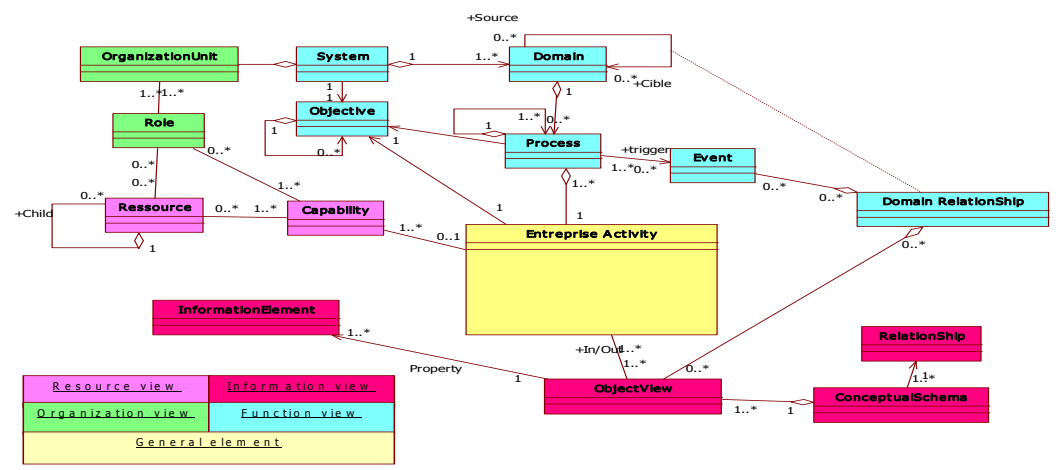

Fig. 4. Meta-model of CIMOSA

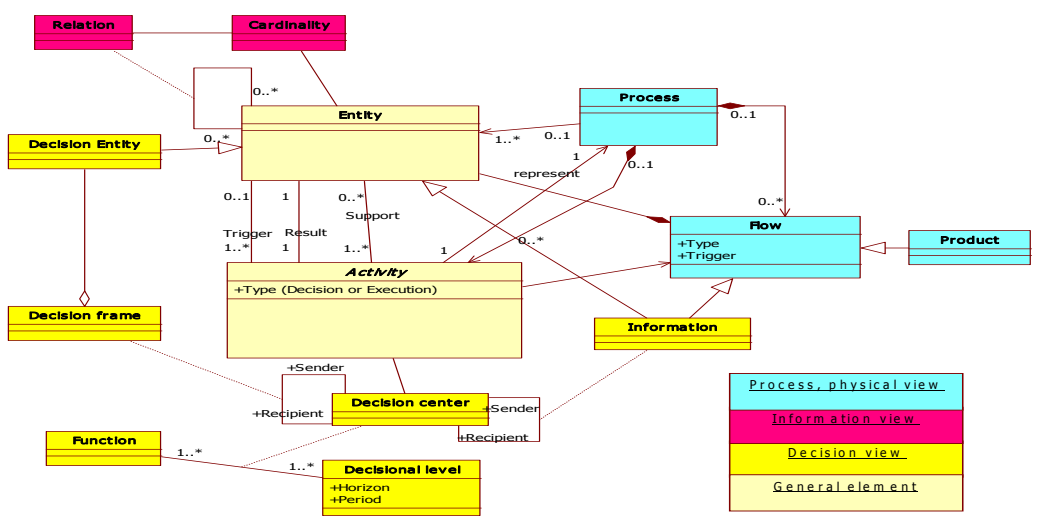

Fig. 5. Meta-model of GRAI/GIM 
GRAI/GIM (GRAI Integrated Methodology) is a methodology for design and analysis of production systems based on the GRAI method [8]. It includes modeling languages and focuses on decision system analysis of the enterprise. In GRAI/GIM, an enterprise consists of a physical system, a decision system and an information system. An enterprise can be described using four views: Functional, Physical, Decisional, and Informational [9]. Fig. 5 illustrates meta-model of GRAI/GIM in class diagram of UML.

\section{Comparison of EM and KM}

Now with a comprehensive look at the methods in modeling both in KM and EM field there are several views. Some of them are common view in all methods like activity and task and some view are unique to each method. Because one of the main objectives of this research is to show and the use the complementary nature of $\mathrm{KM}$ and EM here we propose a generic classification to integrate the modeling views and develop the building block of enterprise knowledge modeling (see Fig. 6).

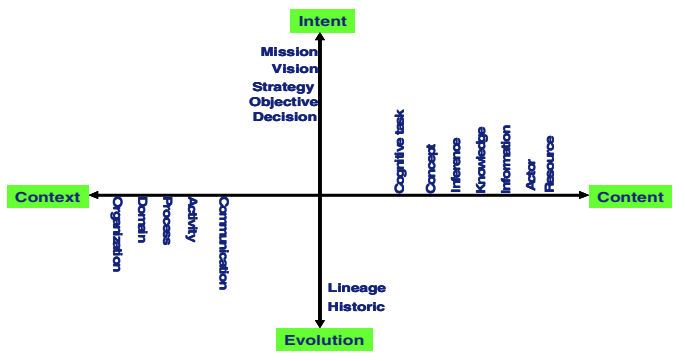

Fig. 6. Views of methods

Both CIMOSA and GRAI/GIM have excellent approach to model organizational context and could provide basic description of operational knowledge context. But they do not provide appropriate explanation of the knowledge being used in the enterprise. Moreover, they do not have any view to model evolution on knowledge and organization. They do not focus on actor or agent directly as a principal source of knowledge. As a matter of intent and intentionality, they provide partial views. For example GRAI/GIM models decision making and CIMOSA partially models the objectives of domain, process, and activities.

Both CommonKADS and MASK provide inclusive views to model knowledge in organization. They provide also advanced approach to content modeling. One weakness of these methods is context modeling. They do not have complete and integrated view to model enterprise context as aforementioned in GRAI/GIM and CIMOSA in meta-model. 


\section{Knowledge Based Enterprise Engineering (KBEE)}

\subsection{KBEE Views}

We propose four generic view of KBEE. These dimensions assist to model knowledge in the organization from multiple points of view;

Context modeling. One of the important issues to model knowledge is to model the context in which knowledge exists. Several methods in both EM and KM develop views and languages to describe the context. This context includes tasks, activities, and business processes in the enterprises. Moreover, the communication view considered as a dimension of context. Although context modeling is the common point of these two fields but one can find more in detail context modeling in EM methods and there are several good languages to model context in EM domain.

Intent modeling. In the literature of both EM and KM, there are several papers that describe the essential of intentionality of top management to launch a global project in the enterprise. However, only in GIM there is a clear decision modeling. Also some sort of organizational modeling in CommonKADS and CIMOSA exist but there is not a clear modeling of strategy and leadership support for the methods. The concepts like mission, vision, and strategy in the enterprises are the source of knowledge and describe global direction of each organization.

Content modeling. Content is the heart of our classification of knowledge modeling. This dimension includes several aspects that should be modeled. Agent modeling is an important view to model knowledge in enterprise. We know that knowledge exists in some form and somewhere in the organization but a very important part of knowledge is tacit knowledge and this knowledge is with the human and so employees in the enterprises. Inference and concept modeling as other important parts of knowledge exist in $\mathrm{KM}$ methods. $\mathrm{KM}$ methods take care of information implicitly in throughout of modeling.

Evolution modeling. Even though in the EM context, the researchers talk about evolution management in the company and maturity models but there is the absence of languages or modeling view dealing with evolution modeling in detail. This aspect is well defiled in MASK methodology in two different models; one for pragmatic dimension as historic modeling and another one for the evolution of sense and signification as lineage modeling.

\subsection{KBEE Meta-model}

Three main elements of KBEE are actor, activity, and artifact (Fig. 7). Artifact includes all elements being used by actor and activity or produced by an activity intended to define what to do. Global objective of the enterprise is the direction of this element. The activity must do by an actor and this actor use the knowledge and some kind of artifact to accomplish that activity. 


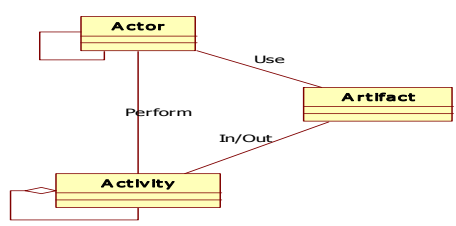

Fig. 7. Three basic components of KBEE

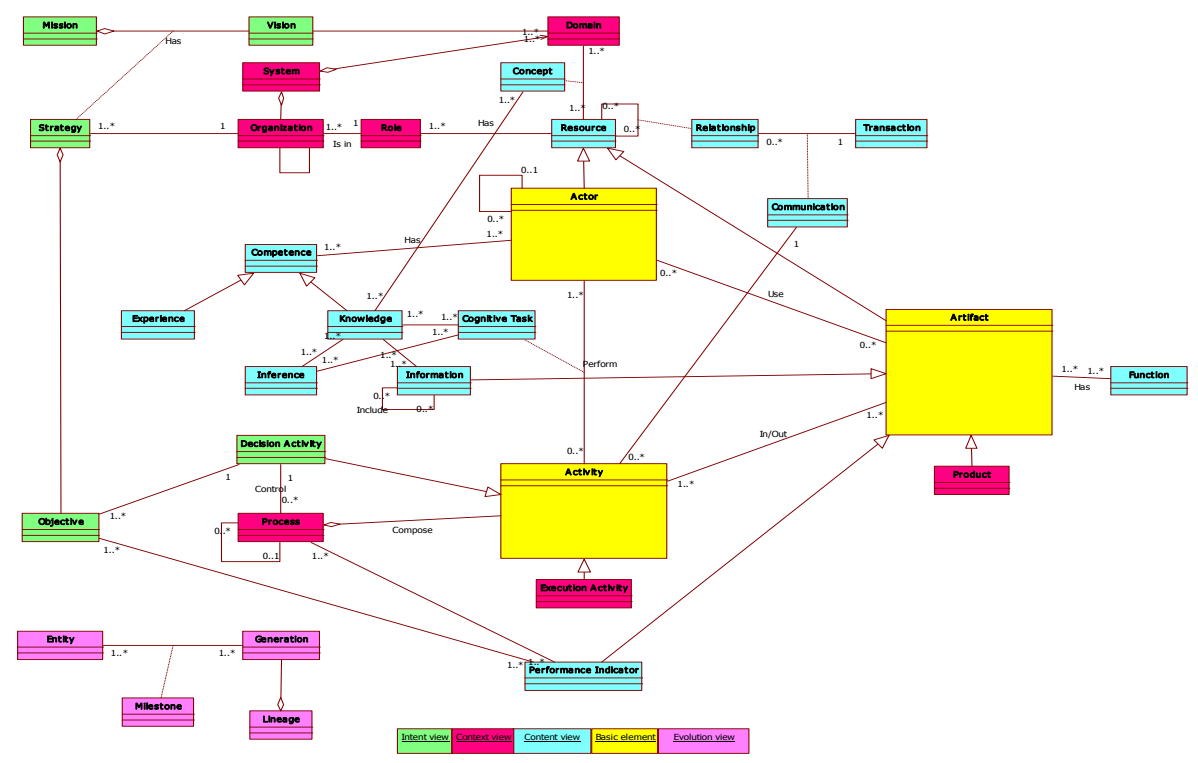

Fig. 8. KBEE meta-model

The elements and concepts being used to KBEE as the results of unification of four methods is described in fig.8. This conceptual meta-model represents essential components of knowledge modeling in the organization. The classed of concepts labeled by green color show the intent concerning concepts. Classes like system, domain, organization and role describe element of context. Communication view and knowledge view in CommonKADS, cognitive task in MASK, and resource view in CIMOSA represent content view in KBEE. Actor, Activity and Artifact here represent the knowledge context for KBEE. Finally evolution view in MASK that originally was intended to model maturity of concepts in the domain is applied to all the classes of KBEE. It means the entire concepts in the context of KBEE will be the object of evolution. To do so, we show evolution as the relation between all the classes that will be subject of evolution.

\section{Conclusion and Perspectives}

The key idea of KM is to provide a way whereby knowledge contents are created, shared, and utilized in an efficient and effective manner. Therefore, it is critical to 
analyze these knowledge requirements. Managers need tools to analyze knowledge contents needed in business processes and decision-making. This paper presents the rationale for knowledge modeling as a foundation for successful KM projects. A method is proposed for building an effective knowledge model. As a modeling language of the method, the unified modeling language (UML) has been chosen.

KBEE to be completed and comprehensive need further works. The formalism and proper languages or tools for the dimension and classed are needed. In this paper we developed the conceptual dimensions of KBEE and we did not enter in the detail level of the KBEE view. Another research direction is to propose the common ontology of concepts, elements and views. How to gather the knowledge and information is the next perspective of this research. This perspective will determine the knowledge sources in the organization, implementation methodology and techniques to collect knowledge in an appropriate way.

\section{References}

1. OMG : Object Management Group, OMG Unified Modeling Language Specification, Version 1.5. (2003), http: / / www . omg . org/docs / formal/ 03-03-01.pdf

2. Schreiber, G., Akkermans, H., Anjewierden, A., de Hoog, R., Shadbolt, N., Van de Velde, W.: Knowledge engineering and management: The Common KADS methodology, 1st edn. The MIT Press, Cambridge (2000)

3. Barthelmé, F., Ermine, J.L., Rosenthal-Sabroux, C.: An architecture for knowledge evolution in organisations. European Journal of Operational Research 109, 414-427 (1998)

4. Ermine, J.L.: La gestion des connaissances, Edition Hermès, Paris (2003)

5. Matta, N., Ermine, J.L., Aubertin, G., Trivin, J.Y.: How to capitalize knowledge with the MASK method? In: IJCAI 2001 Workshop on Knowledge Management and Organizational Memories, Seattle, Washington, USA, August 6 (2001)

6. Vernadat, F.B.: Enterprise modeling and integration: principles and applications. Chapman \& Hall, Boca Raton (1996)

7. Abdmouleh, A., Spadoni, M., Vernadat, F.: Distributed client/server architecture for CIMOSA-based enterprise components. Computers in Industry 55(3), 239-253 (2004)

8. Doumeingts, G., Vallespir, B.: A methodology supporting design and implementation of CIM systems including economic evaluation. In: Brandimarte, P., Villa, A. (eds.) Optimization Models and Concepts in Production Management, pp. 307-331. Gordon \& Breach Science, New York (1995)

9. Vallespir, B., Merle, C., Doumeingts, G.: GIM: a technico-economic methodology to design manufacturing systems. Control Engineering Practice 1(6) (1993) 